\title{
Electrical properties of fluorinated amorphous carbon films
}

\author{
N. Biswas \\ Department of Physics, Texas Tech University, Lubbock, Texas 79409 \\ H. R. Harris \\ Department of Electrical Engineering, Texas Tech University, Lubbock, Texas 79409 \\ X. Wang and G. Celebi \\ Department of Physics, Texas Tech University, Lubbock, Texas 79409 \\ H. Temkin \\ Department of Electrical Engineering, Texas Tech University, Lubbock, Texas 79409 \\ S. Gangopadhyay ${ }^{\mathrm{a})}$ \\ Department of Physics, Texas Tech University, Lubbock, Texas 79409
}

(Received 24 August 2000; accepted for publication 15 January 2001)

\begin{abstract}
We have studied the capacitance-voltage $(C-V)$, conductance-voltage $(G-V)$, and currentvoltage characteristics of fluorinated amorphous carbon $\left(a-\mathrm{C}: \mathrm{F}_{x}\right)$ films using metal $/ a-\mathrm{C}: \mathrm{F}_{x} / \mathrm{Si}$ and metal/ $a-\mathrm{C}: \mathrm{F}_{x} /$ metal structures, respectively. Samples annealed in a vacuum were also studied. The $C-V$ curves of the as-deposited sample are stretched about the voltage axis. Interface state density of $4.1 \times 10^{11} \mathrm{~cm}^{-2} \mathrm{eV}^{-1}$ at the midgap was calculated. Annealing the sample deposited on $\mathrm{Si}$ in a vacuum caused more frequency dispersion in the $C-V$ and $G-V$ curves, probably due to the diffusion of carbon into silicon. The bulk density of states for samples deposited on metal, measured by space-charge-limited current technique, decreased from $4 \times 10^{18} \mathrm{eV}^{-1} \mathrm{~cm}^{-3}$ for the as-deposited sample, to $7 \times 10^{17} \mathrm{eV}^{-1} \mathrm{~cm}^{-3}$ for the annealed sample. (C) 2001 American Institute of Physics.
\end{abstract} [DOI: $10.1063 / 1.1353804]$

\section{INTRODUCTION}

Intense research is being carried out industrywide to find low dielectric constant (low- $\kappa$ ) materials for application in future generation ultralarge scale integrated circuits. Fluorine doped amorphous carbon $\left(a-\mathrm{C}: \mathrm{F}_{x}\right)$ deposited by plasmaenhanced chemical vapor deposition (PECVD) techniques ${ }^{1-3}$ are one of the most promising materials for interlevel dielectrics. These materials are expected to have a dielectric constant as low as 2.0, excellent gap fill properties, ${ }^{4}$ and are relatively simple to implement, compared to spin-on deposited polymers. Various fluorocarbon and hydrocarbon sources are used to deposit these films. We have recently shown that for $a-\mathrm{C}: \mathrm{F}_{x}$ films grown from $\mathrm{CH}_{4}-\mathrm{C}_{4} \mathrm{~F}_{8}$ mixtures at $200 \mathrm{~W}$, postdeposition annealing at $400{ }^{\circ} \mathrm{C}$ leads to reduction of dielectric constant from $2.9 \pm 0.1$ to $2.2 \pm 0.1$. In this article, we present the electrical properties of this film measured by capacitance-voltage $(C-V)$ and current-voltage $(I-V)$ techniques. Since the structural properties of these films depends strongly on process parameters, it is important to correlate the electrical properties of fluorocarbon films with structural properties and investigate how they correlate with process parameters.

The electrical properties of the $a-\mathrm{C}: \mathrm{F}_{x}$ films are best described by the most commonly used metal/insulator/ semiconductor (MIS) structure analysis. Besides the dielectric properties, fixed charge and interface trap density also

a) Author to whom correspondence should be addressed; electronic mail: shubhra.gangopadhyay@ttu.edu require thorough investigation in any dielectric being considered for interlevel dielectric application. Fixed charge is a property of the dielectric material and does not contribute to charge interaction between dielectric and substrate (silicon). Interface traps originate from defects such as dangling bonds at the insulator/substrate interface with energy states in the silicon-forbidden band gap and are dependent on the chemical composition of the interface. ${ }^{5}$ The interface traps can be charged and discharged by varying the applied voltage. These traps can disrupt the distribution of electric field by terminating the electric lines across the $a-\mathrm{C}: \mathrm{F}_{x}$ layer. ${ }^{6}$ It is therefore important to carry out detailed study of the presence of the interface traps. Electrical characterization of the interface by means of capacitance and conductance technique $^{5,7}$ has been proven to be by far the best known. $C-V$, conductance-voltage $(G-V)$, and $I-V$ measurements were used to evaluate $a-\mathrm{C}: \mathrm{F}_{x}$ films deposited on silicon and on silicon coated with TiN/Al/Ti. The metal electrode evaporated on $a-\mathrm{C}: \mathrm{F}_{x} / \mathrm{Si}$ structure comprised of a MIS structure.

\section{EXPERIMENT}

The deposition of $a-\mathrm{C}: \mathrm{F}_{x}$ films was performed in a parallel-plate, capacitively coupled, 13.5 MHz PECVD system (base pressure $10^{-7}$ Torr). Sample A was prepared by placing the substrates on grounded anode at $300{ }^{\circ} \mathrm{C}$, at 200 $\mathrm{W}$ rf power and $600 \mathrm{mTorr}$ gas pressure and sample $\mathrm{B}$ was obtained by annealing sample A at $400^{\circ} \mathrm{C}$ in a vacuum for $30 \mathrm{~min}$. The films were prepared using $\mathrm{CH}_{4}$ and $\mathrm{C}_{4} \mathrm{~F}_{8}$ source 

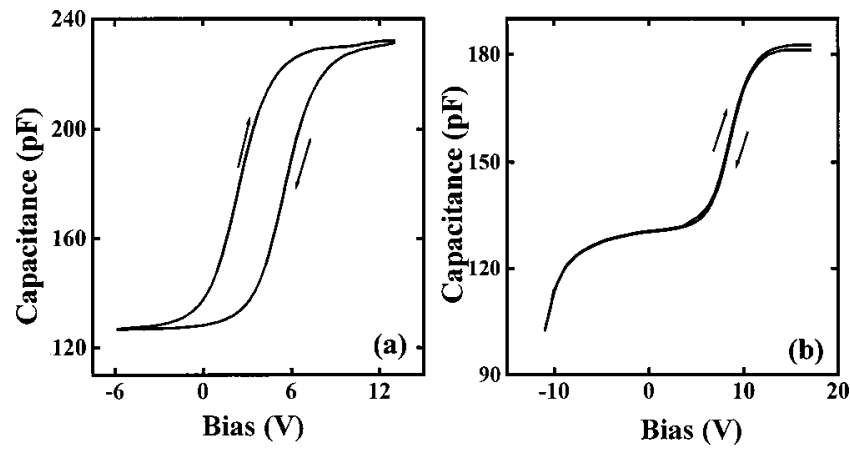

FIG. 1. $C-V$ characteristics showing hysteresis effects for sample A (a) and sample B (b) measured at $10 \mathrm{kHz}$.

gasses on $n$-type crystalline silicon (100) for $C-V, I-V$, and $G-V$ measurements and on $\mathrm{Si}$ coated with TiN/Al/Ti for $I-V$ measurements. The thickness of the film was measured by using a profilometer. Aluminum dots of $1 \mathrm{~mm}$ diameter were vacuum evaporated on these films. Before deposition the silicon wafers were subjected to wet chemical etching. ${ }^{8}$ This process removes the native oxide and produces a hydrogen terminated silicon surface. $C-V$ and $G-V$ measurements were taken on these samples using a HP4275A LCR meter, which has a frequency range from $10 \mathrm{kHz}$ to $10 \mathrm{MHz}$.

The conductance technique involves point-by-point determination of an interface state density throughout the depletion region. The method suggested by Nicollian and Goetzberger $^{7}$ was used to calculate the equivalent parallel conductance. The admittance of a MIS device is measured by a bridge across the diode terminals. A conductance peak is observed because of the ac loss due to capture and emission of carriers by the interface states. From the admittance, the equivalent parallel conductance $\left(G_{p}\right)$ is extracted ${ }^{7}$ which for a single level interface state, characterized by time constant $\tau$, is written as

$$
\frac{G_{p}}{\omega C_{I}}=\frac{q D_{i t} \omega \tau}{\left(1+\omega^{2} \tau^{2}\right) C_{I}},
$$

where $D_{i t}$ is the density of interface states, $C_{I}$ is the insulator capacitance, $q$ is the electronic charge, and $\omega$ is the frequency. When a continuum of interface states is present, this expression then changes to

$$
\frac{G_{p}}{\omega C_{I}}=\frac{q D_{i t}}{2 \omega \tau C_{I}} \ln \left(l+\omega^{2} \tau^{2}\right) .
$$

The maximum value of $G_{p} / \omega C_{I}$ occurs at $\omega \tau=1$ for Eq. (1) and at $\omega \tau=1.98$ for Eq. (2).

\section{RESULTS AND DISCUSSION}

Figures 1(a) and 1(b) show the $C-V$ curves with hysteresis effect of sample A and sample B measured at $10 \mathrm{kHz}$. The thickness of sample A was measured as $1100 \AA$ $\pm 50 \AA$. From the total capacitance of the system (as measured on the $\mathrm{Al} / a-\mathrm{C}: \mathrm{F}_{x} / \mathrm{TiN}$ structure), thickness and contact area $\left(0.0099 \pm 0.01 \mathrm{~cm}^{2}\right)$, we obtained a dielectric con- stant of 2.9 \pm 0.1 for the film. As shown in Fig. 1(a), the constant capacitance after the threshold indicates that inversion has been reached in sample A.

The magnitude of hysteresis for sample A was calculated to be $5 \mathrm{~V}$. The clockwise nature of hysteresis is usually attributed to the trapped charges present in the insulating film, and due to trapping of majority carriers injected from the semiconductor to the insulating film. ${ }^{10,11}$ Such trapping could occur in the insulator if unsaturated bonds and/or vacancies are present. The magnitude of hysteresis was independent of frequency and applied bias. A flatband voltage shift of +6.92 $\mathrm{V}$ was calculated. This indicates the presence of negatively charged ions at the interface. A fixed charge density of $8.68 \times 10^{11} \mathrm{~cm}^{-2}$ was calculated, at a flatband capacitance of $207 \mathrm{pF}$, by comparing the $C-V$ data at $10 \mathrm{kHz}$ with theoretical curve. We have neglected the contribution of work function difference to the flatband voltage shift. This could account for an error of $\sim 15 \%-20 \%$ in the calculation of the aforesaid number. We have also neglected the contribution of mobile ions to the flatband voltage shift since the $C-V$ curves showed no change when the sample was biased up to $20 \mathrm{~V}$.

In the $C-V$ curve for sample B, [Fig. 1(b)], several interesting features can be noted. This includes considerable reduction in hysteresis magnitude, lowering of the maximum capacitance, change in the nature of the $C-V$ curve, and further shift of $C-V$ curve towards positive voltages as compared to sample A. We believe that the reduction in hysteretic effect is due to outgassing of the trapped charges as a result of annealing that leaves pores in the insulating film. The lowering of maximum capacitance can be explained as follows: the pores introduced in the film as a result of annealing causes reduction in mass density of the film which results in expansion of the film and lowers the dielectric constant. $^{12}$ No change in the fluorine concentration was observed after annealing. A 5\% increase in the film thickness was observed a result of annealing. ${ }^{12}$ This was confirmed by grazing incidence $\mathrm{x}$-ray diffraction $(\omega-2 \theta)$ scan, on the asdeposited and annealed samples. Taking into consideration this thickness and the maximum capacitance, as obtained from the relevant $\mathrm{Al} / a-\mathrm{C}: \mathrm{F}_{x} / \mathrm{TiN}$ structure, the dielectric constant was found to be reduced to $2.2 \pm 0.1$. An increase in thickness of $a-\mathrm{C}: \mathrm{F}_{x}$ film, as a result of annealing, has also been observed by several other groups. ${ }^{13,14}$ The pores in the film can also contribute to lowering of maximum capacitance since they might act as capacitors in series with the insulator with air as dielectric.

A change in nature of the $C-V$ curve for sample $\mathrm{B}$, compared to sample $\mathrm{A}$, indicates a deviation from quasiequilibrium conditions after the threshold is reached. Annealing of the sample results in an increase of minority charge carriers that create inversion at the silicon side of the interface, resulting in the plateau seen after the threshold voltage is reached. As the bias is increased, equilibrium inversion conditions are not maintained, since more minority carriers are lost due to injection than are generated and the current through the insulator becomes appreciably large. ${ }^{15}$ At a bias $\sim-7 \mathrm{~V}$, current becomes large enough and is no longer controlled by the insulator but also by the impedance of the 

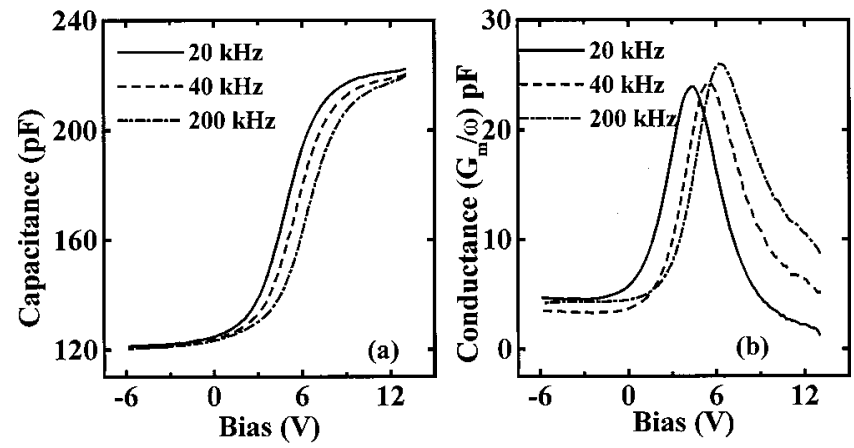

FIG. 2. Capacitance (a) and conductance (b) measurements for sample A.

semiconductor depletion layer. To maintain charge balance, additional donors are depleted, which consequently lowers the semiconductor capacitance by increasing the space charge layer width, resulting in deep depletion of the semiconductor surface.

Figures 2(a) and 2(b) show the frequency dependence of $C-V$ and $G-V$ characteristics for sample A, respectively. As is seen in Fig. 2(a), the $C-V$ curves are stretched about the voltage axis. This is due to the presence of both donor and acceptor like interface traps each occupying a portion of the silicon band gap. ${ }^{16}$ The presence of such traps causes a spread in the $C-V$ curves along the voltage axis. This is commonly referred to as $C-V$ "stretchout." There is no frequency dispersion of capacitance in the accumulation and inversion region. The flatband voltage is frequency dependent and shows frequency dispersion in the depletion region. This is due to the presence of interface traps. At a given frequency, all traps with time constants shorter than the reciprocal of the frequency respond to the measuring signal and the flatband voltage is reached at a particular voltage value. At a higher frequency however, slower traps fail to respond. As a result of which they stay charged; hence the flatband voltage will be reached at a higher gate voltage. The total capacitance of the MIS structure thus is a series combination of the depletion layer capacitance and the capacitance contribution of the frequency dependent interface traps.

Figures 3(a) and 3(b) show the frequency dependence of the $C-V$ and $G-V$ curves of sample B, respectively. Anomalous frequency dispersion of capacitance is observed. The frequency dispersion in accumulation is usually attrib-
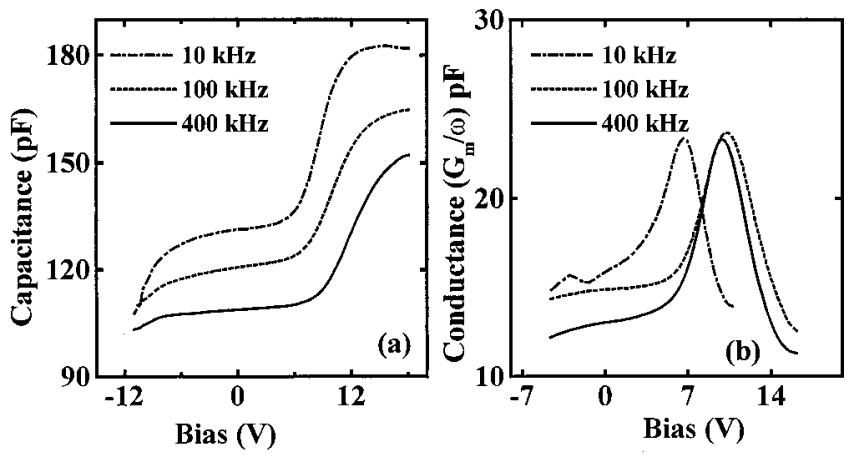

FIG. 3. Capacitance (a) and conductance (b) measurements for sample B.

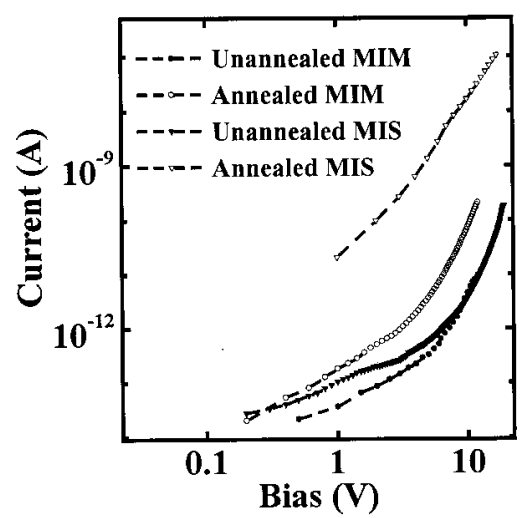

FIG. 4. $I-V$ measurements on $\mathrm{Al} / a-\mathrm{C}: \mathrm{F}_{x} / \mathrm{TiN}$ and $\mathrm{Al} / a-\mathrm{C}: \mathrm{F}_{x}$ structures for sample A and sample B.

uted to the formation of an inhomogeneous layer at the $\mathrm{Si}$ insulator interface. The capacitance of such a layer acts in series with the insulator capacitance causing frequency dispersion of capacitance in the accumulation. There may be an increase in defect states in the insulating layer at the interface. The trapped charges in the defect states account for the increase in $V_{\mathrm{fb}}$ for the annealed sample $\left(V_{\mathrm{fb}} \sim 10.4 \mathrm{~V}\right.$ at 10 $\mathrm{kHz}$ was measured). Formation of an interface layer has also been discussed by several other groups for their MIS systems. ${ }^{17-20}$

The frequency dispersion in depletion region is due to the presence of interface traps as has been explained for the case of sample A. The total capacitance in inversion is increasing with decreasing frequencies. This is because an inversion layer beyond the gate ${ }^{6}$ governs the response time of the minority carriers. This layer beyond the gate is formed due to charges in the insulator. The conducting inversion layer effects an ac current flow along the inversion layer, which spreads laterally over an area that is much larger than the gate area. If the time constants of the minority charge carriers are comparable to the small frequency, an additional capacitance is provided. Frequency dispersion of capacitance in inversion is also observed by Mandel et al. for their diamond like carbon film. ${ }^{21}$

$I-V$ measurements were carried out on samples $\mathrm{A}$ and $\mathrm{B}$, using both $\mathrm{Al} / a-\mathrm{C}: \mathrm{F}_{x} / \mathrm{Si}(\mathrm{MIS})$ and $\mathrm{Al} / a-\mathrm{C}: \mathrm{F}_{x} / \mathrm{TiN}$ [metal-insulator-metal (MIM)] structures. Figure 4 is the plots of $I-V$ measurements for both samples using MIM and MIS structures. For the annealed sample of a MIM structure, there was less than an order of magnitude increase in current observed in both the biasing conditions as compared to the as-deposited sample. This increase in current may be due to conversion of diamond like $s p^{3}$ group to graphite like $s p^{2}$ group, when the sample is annealed. ${ }^{13,14,22}$ The $I-V$ characteristics for the MIM structures consisted of the ohmic part due to the dominance of thermally generated free carriers inside the material at low voltages, and nonohmic spacecharge-limited part due to dominance of injected free carriers at high voltages. In order to check the validity of spacecharge-limited conduction, we verified the scaling $\operatorname{law}^{23,24}$ $J / d=f\left(V / d^{2}\right)$ for $a-\mathrm{C}: \mathrm{F}_{x}$ samples with different thicknesses prepared under same experimental conditions. The density of 

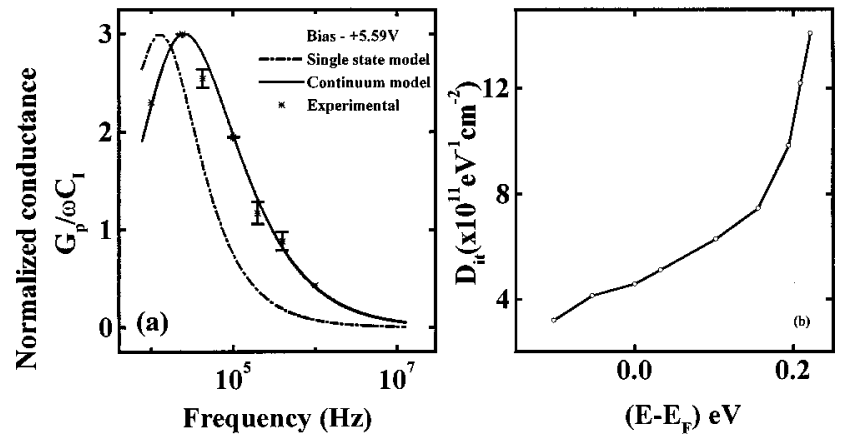

FIG. 5. (a) Normalized equivalent parallel conductance vs frequency for sample A at a gate bias of $+5.59 \mathrm{~V}$ (b) Interface state density vs energy for sample A.

states (DOS) was calculated using the differential method of Nespurek and Sworakowski. ${ }^{25}$ The DOS at the quasi-Fermi level was calculated to be $4 \times 10^{18} \mathrm{eV}^{-1} \mathrm{~cm}^{-3}$ and 7 $\times 10^{17} \mathrm{eV}^{-1} \mathrm{~cm}^{-3}$ for the unannealed and the annealed samples, respectively. This clearly shows that annealing of $a-\mathrm{C}: \mathrm{F}_{x}$ films improves the electrical properties of the bulk material probably due to the outgoing of the trapped radicals.

In the $I-V$ plots for the sample deposited on silicon (MIS structure), there is almost four orders of magnitude increase in current in the annealed sample in both the biasing conditions as compared to the as-deposited sample. We believe that this is due to the chemical reaction between the $\mathrm{Si}$ surface and the carbon due to annealing, that perturbs the electronic distribution and composition at the insulator/Si interface resulting in a relatively higher current flow.

The observed four orders of magnitude increase in current in the annealed sample requires further discussion. A rougher interface can be expected to be present between the insulator and Si for the annealed film. This roughening at the contact results in high field regions, which enhances the injection of carriers and an increased conduction is observed. A possible diffusion of carbon into $\mathrm{Si}$ is suspected causing charge leakage and damaged interface. At $400{ }^{\circ} \mathrm{C}$, however, the diffused carbon probably resides mainly near the silicon surface resulting in $\mathrm{Si}-\mathrm{C}$ bonding. More studies are required to substantiate our hypothesis.

Figure 5(a) is the plot of normalized equivalent parallel conductance versus frequency for the sample A for the specified bias. The curve fitting was done as follows: $G_{p} / \omega C_{I}$ was extracted from the measured capacitance and conductance using the method explained by Nicollian and Goetzberger. ${ }^{7}$ At each fixed bias, the maximum value of $G_{p} / \omega C_{I}$ when plotted against frequency went through a maximum. This value and the corresponding frequency were recorded. These values are then substituted in Eq. (1) to evaluate $D_{\text {it }}$ based on single time constant model and into Eq. (2) to evaluate $D_{\text {it }}$ based on a continuum model. ${ }^{18}$ These values are resubstituted into Eqs. (1) and (2), respectively to generate theoretical $G_{p} / \omega C_{I}$ vs $\log \omega$ plots. The experimental points were then fitted into the plots. Figure 5(a) shows that over the range of bias investigated $G_{p} / \omega C_{I}$ vs $\log \omega$ showed good fit to theory if a continuum of states is as- sumed. We observed the same type of behavior at all values of gate bias in the depletion region.

Figure 5(b) shows a plot of interface state density $\left(D_{\mathrm{it}}\right)$ versus energy for sample A using the continuum of interface states model. $D_{\text {it }}$ for this sample was calculated to be $\sim 4.1$ $\times 10^{11} \mathrm{~cm}^{-2} \mathrm{eV}^{-1}$ at the midgap. A continuum of interface trap levels, as observed in the case of sample A, could result from chemical inhomogeneity at the Si/insulator interface. ${ }^{6}$ The inhomogeneity is caused due to stretched, bent, or broken bonds between the insulator and the Si surface. In our case, such a chemical disorder in the vicinity of the Si surface may be caused from the bonding between $\mathrm{Si}$ and carbon resulting in a stretched or broken bond which causes spreading of interface traps in the Si band gap.

Due to charge leakage, we could not precisely relate bias to surface potential and hence to energy for sample B. The charge leakage in this sample may also result in an inaccurate $D_{\text {it }}$ calculation according to the following explanation. The measured capacitance is directly proportional to the displacement current assuming a constant voltage ramp. ${ }^{10}$ The leakage current adds or subtracts from the displacement current in the dielectric as the bias is varied. Thus, the small signal energy loss is due to interface traps and leakage current. The capacitance measurement could thus be inaccurate leading to incorrect values for $D_{\text {it }}$.

\section{CONCLUSIONS}

The electrical characteristics of as-deposited and annealed $a-\mathrm{C}: \mathrm{F}_{x}$ films have been studied using $C-V, G-V$, and $I-V$ methods. The $C-V$ and $G-V$ curves of the asdeposited sample were shifted and stretched about the voltage axis. The $D_{\text {it }}$ values obtained for this sample are reasonably low from a device point of view. The $I-V$ curves indicate that the as-deposited sample behaves like a perfect insulator and shows reasonably good electrical properties as a low- $\kappa$ dielectric. Electrical properties of the film deposited on $\mathrm{Si}$ changes after annealing, producing additional interface traps and the film tends to be more conductive. A possible diffusion of carbon into silicon may account for damaged interface. The electrical properties of the films deposited on metal improve after annealing at $400{ }^{\circ} \mathrm{C}$ due to the outgassing of trapped radicals. This study shows that it is possible to deposit $a-\mathrm{C}: \mathrm{F}_{x}$ films with low dielectric constant, thermal stability up to $400^{\circ} \mathrm{C}$, and with reasonably low bulk defect density.

\section{ACKNOWLEDGMENTS}

The authors would like to thank Dr. Sergae Nishikin for providing the recipe for cleaning the substrate. Research funds from National Science Foundation under Contract No. ECS-9614593 and State of Texas Advanced Technology Program Grant No. 003644-0229-1999 are gratefully acknowledged.

${ }^{1}$ H. Kudo, S. Takeishi, R. Shinohara, and M. Yamada, Proceedings of the DUMIC Conference, p. 85.

${ }^{2}$ C. B. Labelle, S. J. Limb, and K. K. Gleason, Proceedings of the DUMIC Conference, p. 98. 
${ }^{3}$ H. Yokomichi, T. Hayashi, and A. Masuda, Appl. Phys. Lett. 72, 2704 (1998).

${ }^{4}$ K. Endo, T. Tatsumi, Y. Matsubara, and T. T. Matsubara, Mater. Res. Soc. Symp. Proc. 443, (1996).

${ }^{5}$ S. M. Sze, Physics of Semiconductor Devices, 2nd ed. (Wiley, New York, 1981).

${ }^{6}$ E. H. Nicollian and J. R. Brews, MOS Physics and Technology (Wiley, New York, 1982).

${ }^{7}$ E. H. Nicollian and A. Goetzberger, Bell Syst. Tech. J. 46, 1055 (1967).

${ }^{8}$ S. A. Nikishin, V. G. Antipov, S. Francoeur, N. N. Faleev, G. A. Seryogin, V. A. Elyukhin, and H. Temkin, Appl. Phys. Lett. 75, 484 (1999).

${ }^{9}$ R. F. Pierret, Semiconductor Device Fundamentals (Addison-Wesley, Reading, MA, 1996), p. 597.

${ }^{10}$ D. K. Schroder, Semiconductor Device Theory, 2nd ed. (Wiley, New York, 1998).

${ }^{11}$ F. P. Heiman and G. Warfield, IEEE Trans. Electron Devices 12, 167 (1965).

${ }^{12}$ X. Wang, B. Lahlouh, S. Gangopadhyay, H. R. Harris, and H. Temkin, Proceedings of the VMIC Conference, pp. 187-189.

${ }^{13}$ Hongning Yang, D. J. Tweet, Y. Ma, and T. Nguyen, Appl. Phys. Lett. 73, 1514 (1998).
${ }^{14}$ J. A. Theil, F. Mertz, M. Yairi, K. Seaward, G. Ray, and G. Kooi, Mater. Res. Soc. Symp. Proc. 476, 31 (1997).

${ }^{15}$ F. H. Hielscher and H. M. Preier, Solid-State Electron. 12, 527 (1969).

${ }^{16}$ R. Razouk and B. Deal, J. Electrochem. Soc. 126, 1573 (1979).

${ }^{17}$ H. Shin, M. R. Guire, and A. H. Heuer, J. Appl. Phys. 83, 3311 (1998).

${ }^{18}$ A. A. Khan, J. A. Woollam, Y. Chung, and B. Banks, IEEE Electron Device Lett. 4, (1983).

${ }^{19}$ A. Heime and H. Pagnia, Appl. Phys. 15, 79 (1978).

${ }^{20}$ H. Hasegawa, K. E. Forward, and H. Hartnagel, Appl. Phys. Lett. 26, 567 (1975).

${ }^{21}$ T. Mandel, M. Frischolz, R. Helbig, S. Birkle, and A. Hammerschimdt, Appl. Surf. Sci. 65, 795 (1993).

${ }^{22}$ S. Gangopadhyay, X. Wang, H. R. Harris, G. Celebi, K. Bouldin, D. Lamp, H. Temkin, M. S. Strathman, and M. West, Proceedings of the DUMIC Conference, 1999, pp. 25-30.

${ }^{23}$ M. A. Lampert and P. Mark, Current Injection in Solids (Academic, New York, 1970).

${ }^{24}$ N. Murgatroyd, J. Phys. D 3, 1488 (1970).

${ }^{25}$ S. Nespurek and J. Sworakowski, J. Appl. Phys. 51, 2098 (1980). 